\title{
Acute Exacerbation of Idiopathic Pulmonary Fibrosis Following Treatment for Cushing's Syndrome
}

\author{
Nobumasa Ohara ${ }^{1,2}$, Masanori Kaneko ${ }^{1}$, Kazuhiro Sato ${ }^{3}$, Hiroyuki Usuda ${ }^{4}$, Junta Tanaka ${ }^{5}$, \\ Takashi Maekawa ${ }^{6}$, Hironobu Sasano ${ }^{6}$, Hideki Katakami ${ }^{7}$, Kenzo Kaneko ${ }^{1}$ and Kyuzi Kamoi ${ }^{8}$
}

\begin{abstract}
A 64-year-old Japanese man with mild reticular shadows in both lungs developed a lung tumor causing ectopic Cushing's syndrome. He was prescribed an adrenal inhibitor, which controlled his hypercortisolemia. However, he developed acute exacerbation of idiopathic pulmonary fibrosis (IPF) and died within weeks. Previous studies have suggested a dosage reduction of corticosteroids for IPF as a triggering event for acute exacerbation. The present case suggests that IPF coexisting with Cushing's syndrome may have been exacerbated after the correction of hypercortisolemia. Therefore, close monitoring of cortisol levels along with the clinical course of IPF is required in similar cases that require the correction of hypercortisolemia.
\end{abstract}

Key words: idiopathic pulmonary fibrosis, acute exacerbation, small cell lung carcinoma, ectopic Cushing's syndrome, adrenal inhibitor

(Intern Med 55: 389-394, 2016)

(DOI: 10.2169/internalmedicine.55.5566)

\section{Introduction}

Idiopathic pulmonary fibrosis (IPF), the most frequent form of idiopathic interstitial pneumonia, is a chronic, steadily worsening fibrotic lung disease with no clear etiology, a median survival of 3 years, and few therapeutic options $(1,2)$. Some patients with IPF experience an acute and often fatal respiratory deterioration without any identifiable causes, which is referred to as acute exacerbation of IPF (3).

Endogenous Cushing's syndrome is an endocrine disease that results from chronic exposure to excessive glucocorticoids produced by the adrenal cortex (4). Ectopic adrenocorticotropic hormone syndrome (EAS), a type of Cushing's syndrome caused by adrenocorticotropic hormone (ACTH)secreting non-pituitary tumors, comprises approximately $10 \%$ of endogenous Cushing's syndrome cases. The appropriate management of EAS, when its source is identified, is surgical excision or chemotherapy after the control of hypercortisolemia is obtained using adrenal steroidogenesis inhibitors (5). Although the prognosis of EAS is largely determined by the nature of the underlying tumor, the clinical course can be greatly modified by the control of hypercortisolemia.

We herein report a case of acute exacerbation of IPF that occurred in a patient with small cell lung carcinoma (SCLC), which manifested as EAS.

\section{Case Report}

A 64-year-old Japanese man visited a local hospital in February 2013 with generalized edema of four months' duration. His family history was unremarkable. He had smoked 20 cigarettes per day since he was 26 years of age (38 pack-years smoking). He was found to have reticular shadows in both lungs with a complaint of shortness of

\footnotetext{
${ }^{1}$ Department of Endocrinology and Metabolism, Nagaoka Red Cross Hospital, Japan, ${ }^{2}$ Department of Endocrinology and Metabolism, Uonuma Institute of Community Medicine, Niigata University Medical and Dental Hospital, Japan, ${ }^{3}$ Department of Respiratory Medicine, Nagaoka Red Cross Hospital, Japan, ${ }^{4}$ Department of Pathology, Nagaoka Red Cross Hospital, Japan, ${ }^{5}$ Division of Respiratory Medicine, Graduate School of Medical and Dental Sciences, Niigata University, Japan, ${ }^{6}$ Department of Pathology, Tohoku University School of Medicine, Japan, ${ }^{7}$ Division of Clinical Research Sciences, Department of Medicine, Teikyo University Chiba Medical Center, Japan and ${ }^{8}$ Center of Diabetes, Endocrinology and Metabolism, Joetsu General Hospital, Japan

Received for publication April 11, 2015; Accepted for publication May 27, 2015

Correspondence to Dr. Nobumasa Ohara, oharan@med.niigata-u.ac.jp
} 


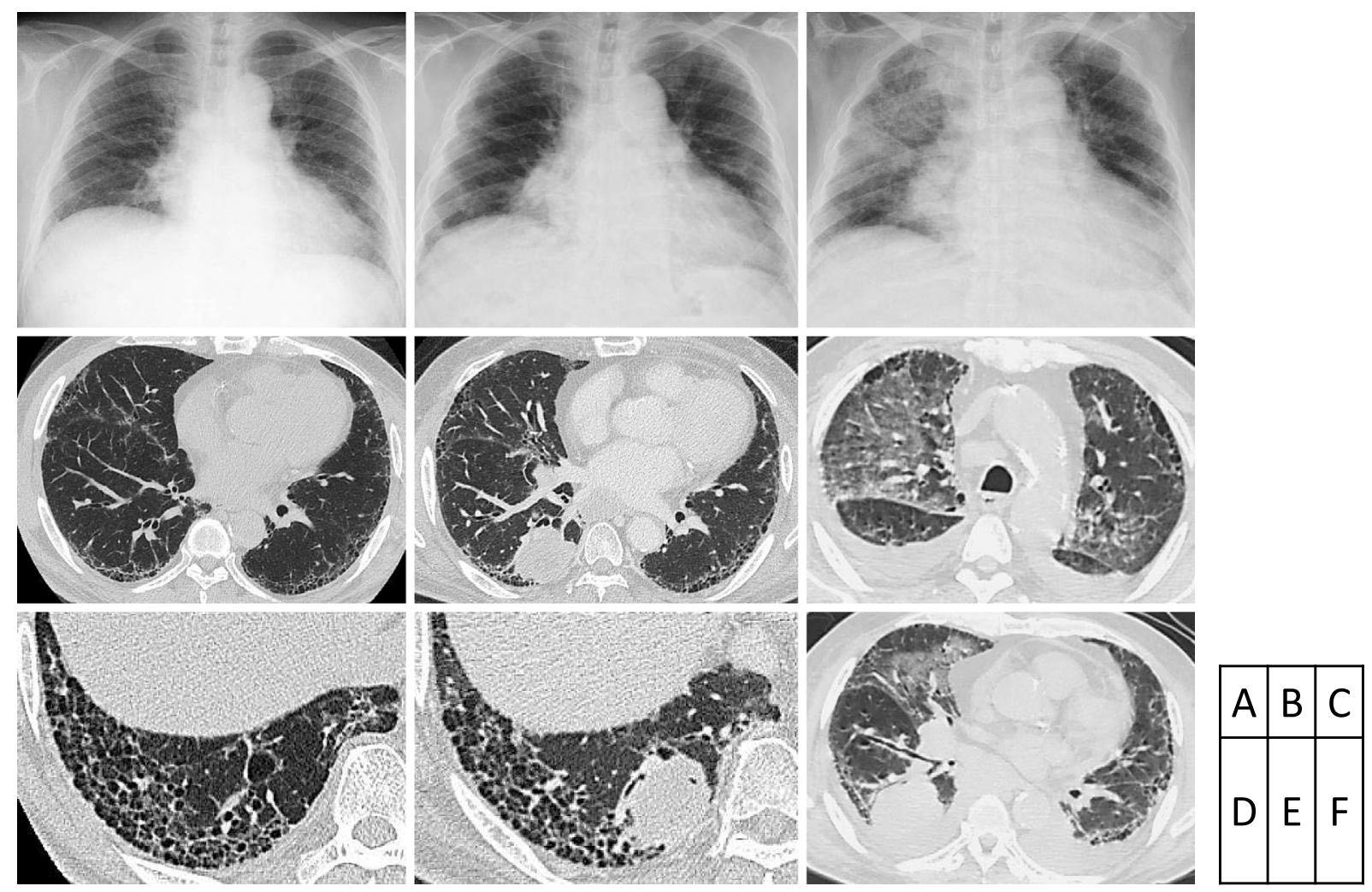

Figure 1. Radiological findings. (A, D) Two years before admission, subtle abnormal shadows that were predominantly located in the lower lungs were detected on a chest $\mathrm{X}$-ray. Chest computed tomography (CT) shows peripheral and basilar predominant reticular abnormalities and subpleural honeycomb changes in both lungs. (B, E) Chest CT 2 weeks before admission and a chest X-ray at the time of admission show the appearance of a $3.5-\mathrm{cm}$ tumor in the right lower lung. The reticular abnormalities and honeycomb changes in both lungs showed almost no changes in comparison with the CT scans that were taken 2 years before admission. (C, F) A chest X-ray and a CT scan on day 11 after admission show the presence of extensive infiltrates in both lungs.

breath at 62 years of age (Fig. 1A, D) and had been followed without medication.

At the hospital visit, the patient's cardiac function was normal, but a chest X-ray and a computed tomography (CT) scan showed a $3.5-\mathrm{cm}$ tumor in the right lower lung (Fig. 1B, E). Swelling of the right hilar and mediastinal lymph nodes was also detected. The reticular shadows in both lungs had remained almost unchanged during the past 2 years. Bronchoscopy was not feasible due to the patient's worsening edema. One month later, he developed hypokalemic periodic paralysis and was admitted to the hospital. He was transferred to our hospital for a further endocrine evaluation in March 2013 because of high serum cortisol levels $(54.5 \mu \mathrm{g} / \mathrm{dL})$ in serum samples taken at $10 \mathrm{AM}$.

The patient presented with a depressed mental status and general weakness, and his performance status (PS) was markedly impaired (Eastern Cooperative Oncology Group PS, 3-4). He was $152 \mathrm{~cm}$ tall and weighed $57 \mathrm{~kg}$. His body temperature, blood pressure, and pulse rate were $36.8^{\circ} \mathrm{C}$, and $160 / 72 \mathrm{mmHg}$, and 86 beats per minute, respectively. No heart murmurs were detected. Mild velcro rales were audible in both lower lungs, with normal percutaneous oxygen saturation $\left(\mathrm{SpO}_{2}\right)$ levels of $95 \%$. He had facial pigmentation, thin skin that was prone to easy bruising, and extreme peripheral edema.

The laboratory findings showed a high white blood cell count, hyperglycemia, hypoproteinemia, and hypokalemia (Table). The serum levels of carcinoembryonic antigen, progastrin releasing peptide, lactate dehydrogenase, and sialylated carbohydrate antigen Krebs von den Lungen-6 were high. An arterial blood gas analysis showed metabolic and respiratory alkalosis and mild hypoxia. Endocrine tests showed ACTH-dependent hypercortisolemia. The patient's urinary free cortisol excretion $(5,615 \mu \mathrm{g} /$ day; reference range, $11.2-80.3 \mu \mathrm{g} /$ day) was extremely high. The overnight oral administration of dexamethasone $(8 \mathrm{mg})$ did not suppress his morning serum cortisol levels $(68.9 \mu \mathrm{g} / \mathrm{dL})$. Magnetic resonance imaging of the brain showed multiple tumors in the cerebrum and pons, but no abnormalities were detected in the pituitary. He was considered to have EAS due to lung cancer with distant metastases.

Because of his poor PS and the lack of a histological diagnosis, anticancer therapy was not indicated for the patient's lung tumor at that point. To improve his Cushing's syndrome symptoms, the oral administration of metyrapone (0.75 g/day) was started on day 6 of admission, and a 
Table. Laboratory Findings at Referral to Our Hospital in March

2013.

\begin{tabular}{|c|c|c|}
\hline \multicolumn{3}{|l|}{ Hematology } \\
\hline Red blood cells & $491 \times 10^{4} / \mu \mathrm{L}$ & $(427-571)$ \\
\hline Hemoglobin & $15.8 \mathrm{~g} / \mathrm{dL}$ & $(12.4-17.2)$ \\
\hline Hematocrit & $46.6 \%$ & $(38.7-50.3)$ \\
\hline White blood cells & $128.2 \times 10^{2} / \mu \mathrm{L}$ & $(40.0-90.0)$ \\
\hline Platelets & $13.0 \times 10^{4} / \mu \mathrm{L}$ & $(12.0-30.0)$ \\
\hline \multicolumn{3}{|l|}{ Chemistry } \\
\hline Total protein & $5.0 \mathrm{~g} / \mathrm{dL}$ & $(6.7-8.3)$ \\
\hline Albumin & $2.8 \mathrm{~g} / \mathrm{dL}$ & $(3.8-5.3)$ \\
\hline Aspartate aminotransferase & $28 \mathrm{IU} / \mathrm{L}$ & $(13-33)$ \\
\hline Alanine aminotransferase & $37 \mathrm{IU} / \mathrm{L}$ & $(8-42)$ \\
\hline Lactate dehydrogenase & $584 \mathrm{IU} / \mathrm{L}$ & $(105-215)$ \\
\hline Urea nitrogen & $17.1 \mathrm{mg} / \mathrm{dL}$ & $(8.0-22.0)$ \\
\hline Creatinine & $0.63 \mathrm{mg} / \mathrm{dL}$ & $(0.6-1.1)$ \\
\hline Sodium & $150 \mathrm{mmol} / \mathrm{L}$ & $(137-147)$ \\
\hline Potassium & $2.9 \mathrm{mmol} / \mathrm{L}$ & $(3.5-4.7)$ \\
\hline Chloride & $109 \mathrm{mmol} / \mathrm{L}$ & $(98-108)$ \\
\hline C-reactive protein & $0.09 \mathrm{mg} / \mathrm{dL}$ & $(<0.30)$ \\
\hline Casual plasma glucose & $12.5 \mathrm{mmol} / \mathrm{L}$ & $(3.9-7.8)$ \\
\hline HbAlc (NGSP) & $9.2 \%$ & $(4.6-6.2)$ \\
\hline Carcinoembryonic antigen & $18.5 \mathrm{ng} / \mathrm{mL}$ & $(<6.0)$ \\
\hline Pro-gastrin releasing peptide & $95.5 \mathrm{pg} / \mathrm{mL}$ & $(<81.0)$ \\
\hline KL-6 & $560 \mathrm{U} / \mathrm{mL}$ & $(<500)$ \\
\hline Anti-nuclear antibody & titer $<1: 40$ & $(<1: 40)$ \\
\hline \multicolumn{3}{|l|}{ Arterial blood gas analysis under room air } \\
\hline $\mathrm{pH}$ & 7.520 & $(7.35-7.45)$ \\
\hline Partial carbon dioxide pressure & $33.0 \mathrm{mmHg}$ & $(32-48)$ \\
\hline Partial oxygen pressure & $71.6 \mathrm{mmHg}$ & $(83-108)$ \\
\hline Bicarbonate & $26.8 \mathrm{mmol} / \mathrm{L}$ & $(21-28)$ \\
\hline Alveolar-arterial oxygen difference & $41.0 \mathrm{mmHg}$ & $(5-10)$ \\
\hline \multicolumn{3}{|l|}{ Endocrinological examination } \\
\hline \multicolumn{3}{|l|}{ Serum cortisol } \\
\hline $8 \mathrm{AM}$ & $62.7 \mu \mathrm{g} / \mathrm{dL}$ & \\
\hline $4 \mathrm{PM}$ & $48.8 \mu \mathrm{g} / \mathrm{dL}$ & \\
\hline 12 PM & $58.6 \mu \mathrm{g} / \mathrm{dL}$ & \\
\hline \multicolumn{3}{|l|}{ Plasma ACTH } \\
\hline $8 \mathrm{AM}$ & $234.8 \mathrm{pg} / \mathrm{mL}$ & \\
\hline $4 \mathrm{PM}$ & $222.8 \mathrm{pg} / \mathrm{mL}$ & \\
\hline $12 \mathrm{PM}$ & $240.2 \mathrm{pg} / \mathrm{mL}$ & \\
\hline
\end{tabular}

prompt reduction was observed in his serum cortisol levels (Fig. 2). The dose of metyrapone was gradually titrated up to $3.5 \mathrm{~g} /$ day, and his serum cortisol levels were maintained at around $20 \mu \mathrm{g} / \mathrm{dL}$.

The patient showed marked improvements in his hypokalemia, diabetes, and hypertension. On day 10 of admission, however, he complained of acute dyspnea. His temperature $\left(36.4^{\circ} \mathrm{C}\right.$ ) was normal, but his $\mathrm{SpO}_{2}$ levels $(91 \%)$ had decreased. His chest X-ray showed infiltrates in the right lung. Acute bacterial pneumonia was initially suspected, and intravenous $1.5 \mathrm{~g} /$ day meropenem was started empirically with oxygen inhalation. However, his respiratory state deteriorated with spreading shadows in both lungs (Fig. 1C, F). Bronchoscopy was performed on day 14 , after an improvement of his severe edema, and SCLC was histologically diagnosed using a biopsy specimen. A bronchoalveolar lavage culture was negative for bacteria and any other pathogens. Acute exacerbation of IPF was considered, and pulse corticosteroid therapy with the intravenous administration of methylprednisolone ( $1 \mathrm{~g} /$ day for 3 days was initiated under mechanical ventilation with bilevel positive airway pressure, while continuing treatment for endogenous Cushing's syndrome. Anticancer therapy was not initiated because of possible pulmonary toxic effects. The pulse methylprednisolone therapy was administered twice, however, the patient's respiratory state worsened, and he died due to respiratory failure 31 days after admission.

A postmortem examination (Fig. 3) showed a mixture of acute and organizing stages of diffuse alveolar damage, which was marked in the relatively preserved lung tissue regions away from the grossly-visible honeycombing structure of the lower lungs. There was no evidence of disseminated metastasis or lymphangitis carcinomatosa. A pattern of usual interstitial pneumonia, such as cystic fibrotic airspaces and fibroblastic foci, was also identified in both of the lower lungs. These pathological findings were consistent with a diagnosis of acute exacerbation of IPF. ACTH-producing SCLC was also confirmed in conjunction with immunohistochemistry. 


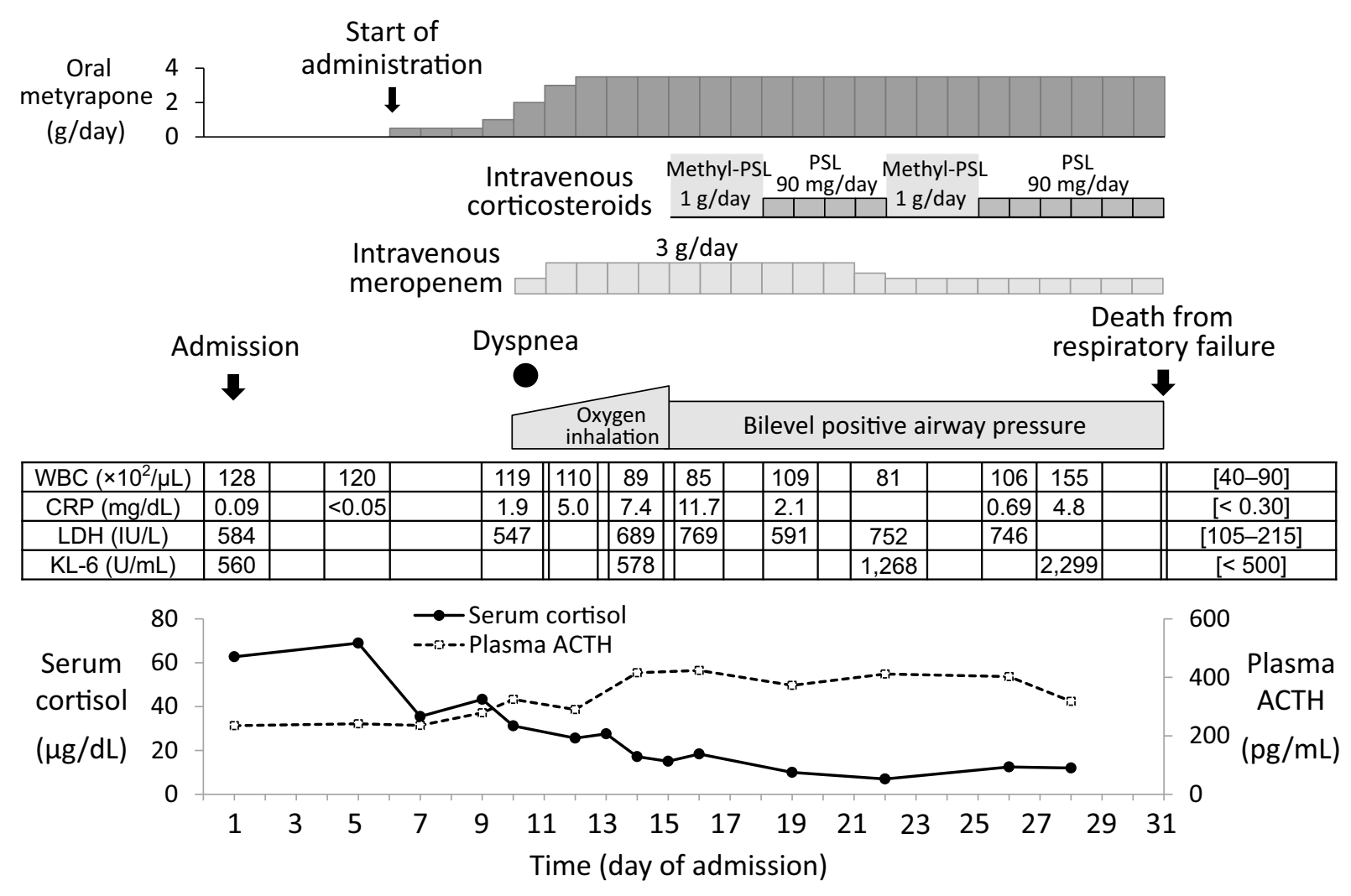

Figure 2. Clinical course. The reference ranges for the white blood cell (WBC) count and the serum levels of C-reactive protein (CRP), lactate dehydrogenase (LDH), and sialylated carbohydrate antigen Krebs von den Lungen-6 (KL-6) are shown in square brackets. ACTH: adrenocorticotropic hormone, PSL: prednisolone

\section{Discussion}

The patient with a two-year history of reticular shadows in both lungs developed ACTH-secreting SCLC with brain metastases manifested by EAS, presenting with severe generalized edema, hypokalemia, diabetes, and hypertension. Because of his poor PS and a lack of histological diagnosis, anticancer therapy was not indicated for his lung cancer at the time of referral to our hospital. After the correction of hypercortisolemia, his Cushing's syndrome symptoms improved. However, the patient suffered a fatal acute respiratory exacerbation and died within weeks of admission. The autopsy findings showed a mixture of acute and organizing diffuse alveolar damage in a background of usual interstitial pneumonia, indicating acute exacerbation of IPF $(1,3)$.

The patient developed acute exacerbation of IPF shortly after his hypercortisolemia was controlled using oral metyrapone, an adrenal steroidogenesis inhibitor (7, 8). Previous studies have suggested a possible role of surgical pulmonary procedures, bronchoscopy, or radiation therapy for lung cancer in the onset of acute exacerbation of IPF (9-12). Our patient had not undergone any of these procedures before his respiratory deterioration. Furthermore, to our knowledge, there have been no reports suggesting the development or deterioration of interstitial pneumonia caused by a drugspecific side effect of metyrapone $(8,12)$. However, a supra- physiological dose of synthetic glucocorticoids was once considered a therapeutic option for idiopathic interstitial pneumonia, including IPF, because of its potential antiinflammatory and immunosuppressive effects $(1,2)$. In addition, several studies have suggested that a dosage reduction of synthetic glucocorticoids for idiopathic interstitial pneumonia is a triggering factor for acute exacerbation (13). The present case suggests that IPF coexisting with endogenous Cushing's syndrome can be exacerbated upon the control of hypercortisolemia. Therefore, the close monitoring of cortisol levels along with the clinical course of IPF is needed in similar cases of Cushing's syndrome that require the correction of hypercortisolemia. In addition, because there is currently no proven therapy for preventing acute exacerbation in patients with IPF $(1,14)$, the non-treatment of hypercortisolemia might be another therapeutic option for patients with similar conditions.

The clinical outcome of acute exacerbation of IPF is generally poor, with a short-term mortality rate of more than $50 \%$, despite therapy. The present treatment strategies lack standardization, and empirical therapy with high-dose synthetic glucocorticoids is commonly used despite it having no proven benefit $(1,3)$. Our patient received 2 courses of pulse corticosteroid therapy for exacerbated IPF while treatment for endogenous Cushing's syndrome was continued, but he failed to respond to the therapy and died from respiratory failure. 

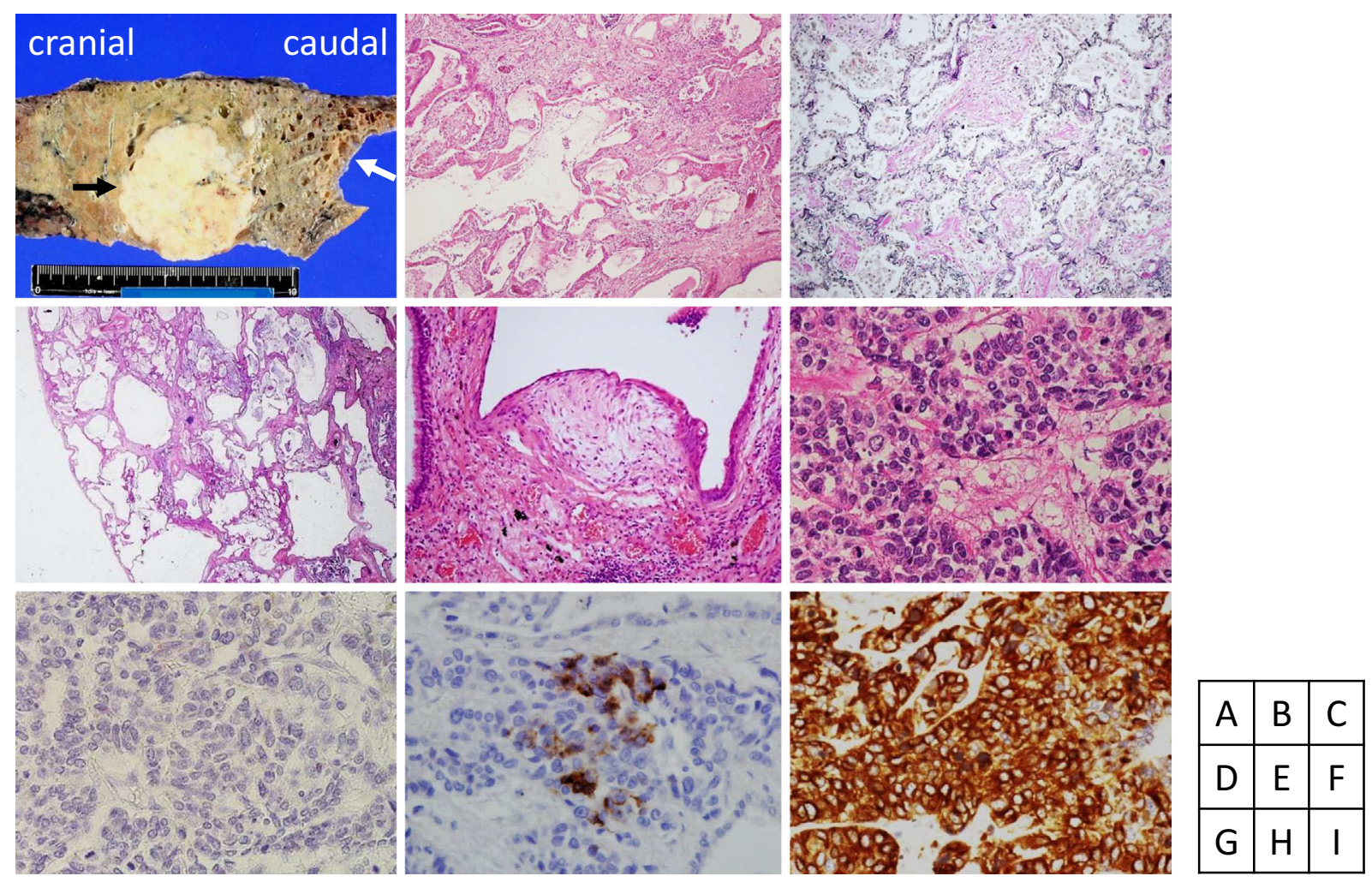

Figure 3. Autopsy findings in both lungs. (A) The gross appearance of the cut surface of the right lower lung shows a 5-cm tumor (black arrow) and a honeycomb-like structure (white arrow). (B-E) The microscopic examination of non-tumoral tissues of the lungs. Hyaline membranes were observed lining the alveoli [(B), Hematoxylin and Eosin (H\&E) staining] and organizing foci with collagenous fibers are seen in the alveoli [(C), Elastica-van Gieson staining] throughout the lungs. These changes, which are consistent with acute and organizing diffuse alveolar damage, were marked in the areas of relatively preserved lung tissue, away from the honeycombing of the lower lungs. No evidence of disseminated metastasis or lymphangitis carcinomatosa was found. In the honeycomb regions of the lower lungs, cystic fibrotic airspaces [(D), H\&E staining, low magnification] and a number of fibroblastic foci [(E), H\&E staining, high magnification] were observed. (F-I) The microscopic examination of the lung tumor cells showed an increased nuclear/cytoplasmic ratio, nuclear fragility, and deoxyribonucleic acid staining [(F), H\&E staining]. No tumor cells showed corticotropin-releasing hormone immunostaining (G), but some were positive for adrenocorticotropic hormone (H), and most were positive for $\gamma 3$-melanocyte stimulating hormone, a proopiomelanocortin-derived peptide (I).

In summary, we herein described a fatal case of acute exacerbation of IPF after the treatment of hypercortisolemia in a patient with advanced ACTH-secreting SCLC, who presented with EAS. The outcome of the present case suggests that IPF coexisting with endogenous Cushing's syndrome may be exacerbated after the treatment of hypercortisolemia. The close monitoring of cortisol levels along with the clinical course of IPF is needed in similar cases of Cushing's syndrome that require the correction of hypercortisolemia. The non-treatment of hypercortisolemia might be another acceptable therapeutic option for patients with similar conditions.

The authors state that they have no Conflict of Interest (COI).

\section{Acknowledgement}

We thank the medical laboratory technicians of Nagaoka Red Cross Hospital for their helpful technical support. We also thank Dr. Hirohito Sone and Dr. Eiichi Suzuki (Niigata University, Japan) for their excellent advice.

\section{References}

1. Raghu G, Collard HR, Egan JJ, et al; ATS/ERS/JRS/ALAT Committee on Idiopathic Pulmonary Fibrosis. An official ATS/ERS/ JRS/ALAT statement: idiopathic pulmonary fibrosis: evidencebased guidelines for diagnosis and management. Am J Respir Crit Care Med 183: 788-824, 2011.

2. Cottin V. Interstitial lung disease. Eur Respir Rev 22: 26-32, 2013.

3. Antoniou KM, Wells AU. Acute exacerbations of idiopathic pulmonary fibrosis. Respiration 86: 265-274, 2013.

4. Newell-Price J, Bertagna $X$, Grossman AB, Nieman LK. Cushing's syndrome. Lancet 367: 1605-1617, 2006. 
5. Alexandraki KI, Grossman AB. The ectopic ACTH syndrome. Rev Endocr Metab Disord 11: 117-126, 2010.

6. Kashiwagi A, Kasuga M, Araki E, et al; Committee on the Standardization of Diabetes Mellitus-Related Laboratory Testing of Japan Diabetes Society. International clinical harmonization of glycated hemoglobin in Japan: From Japan Diabetes Society to National Glycohemoglobin Standardization Program values. J Diabetes Invest 3: 39-40, 2012.

7. Díez JJ, Iglesias P. Pharmacological therapy of Cushing's syndrome: drugs and indications. Mini Rev Med Chem 7: 467-480, 2007.

8. Schteingart DE. Drugs in the medical treatment of Cushing's syndrome. Expert Opin Emerg Drugs 14: 661-671, 2009.

9. Yüksel M, Ozyurtkan MO, Bostanci K, Ahiskali R, Kodalli N. Acute exacerbation of interstitial fibrosis after pulmonary resection. Ann Thorac Surg 82: 336-338, 2006.

10. Hyzy R, Huang S, Myers J, Flaherty K, Martinez F. Acute exacer- bation of idiopathic pulmonary fibrosis. Chest 132: 1652-1758, 2007.

11. Nagano T, Kotani Y, Fujii O, et al. A case of acute exacerbation of idiopathic pulmonary fibrosis after proton beam therapy for non-small cell lung cancer. Jpn J Clin Oncol 42: 965-969, 2012.

12. Camus P, Fanton A, Bonniaud P, Camus C, Foucher P. Interstitial lung disease induced by drugs and radiation. Respiration 71: 301326, 2004.

13. Kondo A, Saiki S. Acute exacerbation in idiopathic interstitial pneumonia (IIP). In: Japan Intractable Diseases Research Foundation Publication No. 27. Interstitial Pneumonia of Unknown Etiology. Harasawa M, Fukuchi Y, Morinari H, Eds. University of Tokyo Press, Tokyo, 1989: 33-42.

14. Ahluwalia N, Shea BS, Tager AM. New therapeutic targets in idiopathic pulmonary fibrosis. Aiming to rein in runaway woundhealing responses. Am J Respir Crit Care Med 190: 867-878, 2014.

(C) 2016 The Japanese Society of Internal Medicine http://www.naika.or.jp/imonline/index.html 